TRANSACTIONS OF THE

AMERICAN MATHEMATICAL SOCIETY

Volume 348, Number 6, June 1996

\title{
COMPOSITION OPERATORS BETWEEN BERGMAN AND HARDY SPACES
}

\author{
WAYNE SMITH
}

\begin{abstract}
We study composition operators between weighted Bergman spaces. Certain growth conditions for generalized Nevanlinna counting functions of the inducing map are shown to be necessary and sufficient for such operators to be bounded or compact. Particular choices for the weights yield results on composition operators between the classical unweighted Bergman and Hardy spaces.
\end{abstract}

\section{INTRODUCTION}

Let $D$ be the open unit disk in the complex plane and denote Lebesgue measure on $D$ by $d A$, normalized so that $A(D)=1$. For $p>0$, the Hardy space $H^{p}$ is the space of functions $f$ that are analytic on $D$ and satisfy

$$
\|f\|_{H^{p}}^{p}=\lim _{r \rightarrow 1-} \frac{1}{2 \pi} \int_{0}^{2 \pi}\left|f\left(r e^{i \theta}\right)\right|^{p} d \theta<\infty,
$$

and the Bergman space $A_{p}$ consists of those analytic functions such that

$$
\|f\|_{A^{p}}^{p}=\int_{D}|f|^{p} d A<\infty .
$$

It is clear that if $p \leq q$, then $H^{q} \subset H^{p}$ and $A^{q} \subset A^{p}$, and also $H^{p} \subset A^{p}$ for all $p$. Moreover, (when $p<q$ ) each of these inclusions is proper. Finally, $H^{\infty}$ is the space of bounded analytic functions on $D$, with $\|f\|_{H^{\infty}}=\sup \{|f(z)|: z \in D\}$. Then $H^{\infty} \subset H^{p}$ for all $p$, and this inclusion is proper if $p<\infty$.

Let $\varphi: D \rightarrow D$ be an analytic self-map of $D$. It is a well known consequence of Littlewood's subordination principle $[\mathrm{L}]$ that $\varphi$ induces through composition a bounded linear operator on the classical Hardy and Bergman spaces (see for example [MS], [Sh] or [Sh2]). That is, if we define $C_{\varphi}$ by $C_{\varphi}(f)=f \circ \varphi$, then $C_{\varphi}: H^{p} \rightarrow H^{p}$ and $C_{\varphi}: A^{p} \rightarrow A^{p}$ are bounded operators. Such operators are called composition operators. Our goal here is to characterize those analytic functions $\varphi: D \rightarrow D$ that induce bounded composition operators from one Bergman or Hardy space to another such space.

As a simple first case, consider the problem of characterizing those $\varphi$ that induce a bounded composition operator from $A^{p}$ to $H^{\infty}$. It is well known (see $\S 4$ ) that

Received by the editors February 23, 1995.

1991 Mathematics Subject Classification. Primary 47B38; Secondary 30D55, 46E15.

Key words and phrases. Bergman spaces, Hardy spaces, composition operators, Nevanlinna counting function.

(C)1996 American Mathematical Society 
if $p<\infty$ and $\left|z_{n}\right| \rightarrow 1-$, then there are $f_{n} \in A^{p}$ such that $\left\|f_{n}\right\|_{A^{p}}=1$ and $\left|f_{n}\left(z_{n}\right)\right| \rightarrow \infty$. Thus $\|\varphi\|_{H^{\infty}}<1$ is necessary. This is also sufficient, since, by the Closed Graph Theorem, we just need to check that $C_{\varphi}(f) \in H^{\infty}$ for every $f \in A^{p}$. Thus the answer in this simple first case is that $C_{\varphi}: A^{p} \rightarrow H^{\infty}$ is bounded if and only if $\varphi(D)$ is a relatively compact subset of $D$, and similar considerations provide the same answer for $C_{\varphi}: H^{p} \rightarrow H^{\infty}$.

This suggests that the general problem, characterizing those $\varphi$ that induce bounded composition operators from $H^{p}$ or $A^{p}$ to $H^{q}$ or $A^{q}$, should have a solution that in some way expresses that $\varphi(D)$ is a small subset of $D$. This turns out to be correct, but not in the literal sense that the image of $D$ under $\varphi$ is a small subset of $D$. Indeed, we will see in $\S 6$ that there is a univalent $\varphi$ such that $C_{\varphi}: A^{p} \rightarrow H^{q}$ is bounded for all $p>0$ and all $q<\infty$, and yet $\overline{\varphi(D)}=\bar{D}$. Our solution involves a measure of how often, and where, $\varphi$ assumes values near the unit circle.

The classical Nevanlinna counting function for $\varphi, N_{\varphi}$, provides such a measure. It is defined by

$$
N_{\varphi}(w)=\sum_{z \in \varphi^{-1}\{w\}} \log (1 /|z|), \quad w \in D \backslash\{\varphi(0)\} .
$$

As is usual, $z \in \varphi^{-1}\{w\}$ is repeated according to the multiplicity of the zero of $\varphi-w$ at $z$. The counting function $N_{\varphi}$ was first used to study composition operators by J. H. Shapiro in [Sh], where he used $N_{\varphi}$ to give a formula for the essential norm of the composition operator from $H^{2}$ to $H^{2}$ induced by $\varphi$. In that paper Shapiro also introduced the generalized counting functions $N_{\varphi, \gamma}$, defined for $\gamma>0$ by

$$
N_{\varphi, \gamma}(w)=N_{\gamma}(w)=\sum_{z \in \varphi^{-1}\{w\}}[\log (1 /|z|)]^{\gamma}, \quad w \in D \backslash\{\varphi(0)\},
$$

and used them to study composition operators from a weighted Bergman space to itself. Thus $N_{\varphi, 1}$ is the Nevanlinna counting function. As indicated above, $N_{\gamma}$ will also be used to denote $N_{\varphi, \gamma}$ when there is no possibility of confusion and no reason to emphasize the dependence on $\varphi$. Here we use these generalized counting functions to characterize those $\varphi$ that induce bounded and compact composition operators between Bergman and Hardy spaces. Before stating our results, we briefly describe previous results of this type.

The problem of characterizing $\varphi$ such that $C_{\varphi}: H^{p} \rightarrow H^{q}$ has been considered by several authors, beginning with $\mathrm{H}$. Hunziker and H. Jarchow in [HJ] (see also $[\mathrm{H}])$. In this paper they observed that, for $\eta \geq 1, C_{\varphi}: H^{p} \rightarrow H^{\eta p}$ is bounded for some $p>0$ if and only if it is bounded for all $p>0$. They then characterized those $\varphi$ that induce such composition operators as those for which $m_{\varphi}$ satisfies an $\eta$-Carleson measure condition, where $m_{\varphi}$ is the image measure of Lebesgue measure on $\partial D$ under the Fatou extension of $\varphi$ to $\partial D$ (see Theorem 3.1 in [HJ]). That is, if $\varphi^{*}: \partial D \rightarrow \bar{D}$ is defined by taking radial limits of $\varphi$, then $m_{\varphi}(A)=m\left(\left(\varphi^{*}\right)^{-1}(A)\right)$ for all Borel sets $A \subset \bar{D}$, where $m$ is Lebesgue measure on $\partial D$. Then R. Riedl, making use of this result, recently proved the following theorem in his dissertation.

Theorem A [R, Theorem IV.3 and Theorem IV.4]. Let $0<p \leq q$ and suppose $\varphi$ is an analytic self-map of $D$. Then $C_{\varphi}: H^{p} \rightarrow H^{q}$ is bounded if and only if

$$
N_{\varphi, 1}(w)=O\left([\log (1 /|w|)]^{q / p}\right) \quad(|w| \rightarrow 1),
$$


and moreover $C_{\varphi}$ is compact if and only if

$$
N_{\varphi, 1}(w)=o\left([\log (1 /|w|)]^{q / p}\right) \quad(|w| \rightarrow 1)
$$

Recall that an operator is said to be compact provided it takes bounded sets to sets with compact closure. This theorem is an example of a recurring theme in operator theory: If a "big-oh" condition determines when an operator is bounded, then the corresponding "little-oh" condition determines when it is compact. We will see several more examples of this. In the upper bounds for $N_{\varphi, 1}$ in Theorem A and in the theorems below, $\log (1 /|w|)$ can be freely replaced by $1-|w|$, since these are comparable as $|w| \rightarrow 1$.

The approach used here does not depend on the results in [HJ] and $[R]$, and yields a different proof of Theorem A. We now state the main results of this paper.

1.1 Theorem. Let $0<p \leq q$ and suppose $\varphi$ is an analytic self-map of $D$. Then

a) $C_{\varphi}: A^{p} \rightarrow A^{q}$ is bounded if and only if

$$
N_{\varphi, 2}(w)=O\left([\log (1 /|w|)]^{2 q / p}\right) \quad(|w| \rightarrow 1)
$$

b) $C_{\varphi}: A^{p} \rightarrow H^{q}$ is bounded if and only if

$$
N_{\varphi, 1}(w)=O\left([\log (1 /|w|)]^{2 q / p}\right) \quad(|w| \rightarrow 1)
$$

c) $C_{\varphi}: H^{p} \rightarrow A^{q}$ is bounded if and only if

$$
N_{\varphi, 2}(w)=O\left([\log (1 /|w|)]^{q / p}\right) \quad(|w| \rightarrow 1) .
$$

Moreover, in each case $C_{\varphi}$ is compact if and only if the corresponding "little-oh" condition is satisfied.

The correspondence between the bounds for $N_{\varphi, 1}$ in Theorem A and Theorem $1.1 \mathrm{~b}$ ), and the bounds for $N_{\varphi, 2}$ in parts a) and c) of Theorem 1.1 immediately yields the following corollaries.

1.2 Corollary. Let $\eta \geq 2$ and suppose $\varphi$ is an analytic self-map of $D$. Then the following are equivalent.

a) There exists $p>0$ such that $C_{\varphi}: H^{p} \rightarrow H^{\eta p}$ is bounded;

b) $C_{\varphi}: H^{p} \rightarrow H^{\eta p}$ is bounded for all $p>0$;

c) There exists $p>0$ such that $C_{\varphi}: A^{p} \rightarrow H^{\eta p / 2}$ is bounded;

d) $C_{\varphi}: A^{p} \rightarrow H^{\eta p / 2}$ is bounded for all $p>0$.

Moreover, these four statements remain equivalent when "bounded" is replaced by "compact".

Remark. The equivalence of parts a) and b) of Corollary 1.2 just requires that $\eta \geq 1$, as is clear from Theorem A and, as noted before, was observed by Hunziker and Jarchow $[\mathrm{HJ}]$. We need $\eta \geq 2$ to assure that the hypothesis $q=\eta p / 2 \geq p$ of Theorem 1.1 is satisfied. 
1.3 Corollary. Let $\eta \geq 1$ and suppose $\varphi$ is an analytic self-map of D. Then the following are equivalent.

a) There exists $p>0$ such that $C_{\varphi}: A^{p} \rightarrow A^{\eta p}$ is bounded;

b) $C_{\varphi}: A^{p} \rightarrow A^{\eta p}$ is bounded for all $p>0$;

c) There exists $p>0$ such that $C_{\varphi}: H^{p} \rightarrow A^{2 \eta p}$ is bounded;

d) $C_{\varphi}: H^{p} \rightarrow A^{2 \eta p}$ is bounded for all $p>0$.

Moreover, these four statements remain equivalent when "bounded" is replaced by "compact".

There also is an elementary relationship between $N_{\varphi, 1}$ and $N_{\varphi, 2}$ : If $w \in$ $D \backslash\{\varphi(0)\}$, then

$$
N_{\varphi, 2}(w)=\sum_{z \in \varphi^{-1}\{w\}}[\log (1 /|z|)]^{2} \leq\left(\sum_{z \in \varphi^{-1}\{w\}} \log (1 /|z|)\right)^{2}=\left(N_{\varphi, 1}(w)\right)^{2} .
$$

The next corollary is an immediate consequence of this, Theorem A and Theorem $1.1 \mathrm{a})$.

1.4 Corollary. Let $\eta \geq 1$ and suppose $\varphi$ is an analytic self-map of D. If $C_{\varphi}$ : $H^{p} \rightarrow H^{\eta p}$ is bounded for some (and hence all) $p>0$, then $C_{\varphi}: A^{p} \rightarrow A^{\eta p}$ is bounded for all $p>0$. Moreover, this remains true when "bounded" is replaced by "compact".

As was noted earlier, when $\eta=1$ parts a) and b) of Corollary 1.3 hold for every analytic self-map $\varphi$ of $D$, as a consequence of Littlewood's subordination principle. Hence $C_{\varphi}: H^{p} \rightarrow A^{2 p}$ is bounded for all $p>0$ and for every $\varphi$ as well. This can also be seen directly. Indeed, using Hardy's inequality [D, p. 48] it is easy to show that $H^{1} \subset A^{2}$, and then $H^{p} \subset A^{2 p}$ for all $p$ follows by using the standard inner-outer factorization of a function in $H^{p}$. Thus $C_{\varphi}: H^{p} \rightarrow A^{2 p}$ is a consequence of $C_{\varphi}: A^{2 p} \rightarrow A^{2 p}$, which, as previously noted, follows from Littlewood's subordination principle.

From these observations, we see that Theorem $1.1 \mathrm{c}$ ) only says something of interest when $q>2 p$; if $q \leq 2 p$, then $C_{\varphi}: H^{p} \rightarrow A^{2 p} \subset A^{q}$ and the statements in Theorem $1.1 \mathrm{c}$ ) are satisfied for every $\varphi$. Similarly, the statements in Theorem 1.1 a) are always valid when $q \leq p$, since $A^{p} \subset A^{q}$ in this case, and once again $C_{\varphi}: A^{q} \rightarrow A^{q}$ is bounded for every analytic self-map of $D$.

In Theorem $1.1 \mathrm{~b}$ ), however, the requirement that $q \geq p$ can not be dropped. To see this, set $p=2 q$ and $\varphi(z) \equiv z$. Then $N_{\varphi, 1}(w)=\log (1 /|w|)$, so that the upper bound for $N_{\varphi, 1}$ in Theorem $1.1 \mathrm{~b}$ ) holds. For this $\varphi, C_{\varphi}$ is the identity map. Since all functions in $H^{q}$ are of bounded characteristic while $A^{p}$ contains functions which are not, it follows that $C_{\varphi}$ does not map $A^{p}$ into $H^{q}$. Thus the sufficient condition in Theorem $1.1 \mathrm{~b}$ ) for $C_{\varphi}$ to be bounded can not be valid for all $p$ and $q$, although it will be shown in a later section that the condition is always necessary. The next theorem provides a sufficient condition for boundedness of $C_{\varphi}$ when $q<p$ that agrees with the condition in Theorem $1.1 \mathrm{~b}$ ) in the limit as $q$ increases to $p$.

1.5 Theorem. Let $\varphi$ be an analytic self-map of $D$, let $0<q<p$, and suppose there is a constant $\eta>(p+q) / p$ such that

$$
N_{\varphi, 1}(w)=O\left([\log (1 /|w|)]^{\eta}\right) \quad(|w| \rightarrow 1) .
$$


Then $C_{\varphi}: A^{p} \rightarrow H^{q}$ is bounded, and moreover is compact.

The similarity of the statements of Theorem A and the various parts of Theorem 1.1 suggests that they are special cases of a more general theorem. This is indeed the case, and it is in this greater generality that they will be proved in the following sections of the paper.

In the next section we introduce a family of weighted Bergman spaces and provide some background material and properties of them. Then, in $\S 3$, we prove our main results giving sufficient conditions for $\varphi$ to induce a bounded composition operator between these spaces. Section 4 is devoted to necessary conditions for $C_{\varphi}$ to be bounded. Corollary 4.4 at the end of $\S 4$ combines the main results of that section and $\S 3$, thereby characterizing those analytic self-maps of $D$ that induce bounded composition operators between certain weighted Bergman spaces. The question of compactness of $C_{\varphi}$ is taken up in $\S 5$. Theorem 5.1, in particular, is the "littleoh" / compactness version of Corollary 4.4. Theorem A and the various parts of Theorem 1.1 are all special cases of the combination of Corollary 4.4 and Theorem 5.1. Similarly, Theorem 1.5 is a special case of Theorem 3.2 and Theorem 5.2. We then end with some applications and examples in $\S 6$.

The approach to the problems considered in this paper comes from J. H. Shapiro's work on the compactness problem for $C_{\varphi}: H^{2} \rightarrow H^{2}$ and his formula for the essential norm of this operator in [Sh]. See also Chapter 10 of [Sh2] for an accessible exposition of the ideas involved in that work.

\section{BACKGround MATERIAL}

2.1 Weighted Bergman spaces. We introduce a family of weighted Bergmantype spaces that allows us to handle the classical Bergman and Hardy spaces in a unified manner. For $\gamma>-1$ define the measure $d A_{\gamma}$ on $D$ by

$$
d A_{\gamma}(w)=[\log (1 /|w|)]^{\gamma} d A(w)
$$

For $0<p<\infty$ and $\gamma>-1$ we define the weighted Bergman space $A_{\gamma}^{p}$ to be those functions $f$ analytic on $D$ and satisfying

$$
\|f\|_{A_{\gamma}^{p}}^{p}=\int_{D}|f(w)|^{p} d A_{\gamma}(w)<\infty .
$$

In this definition, the measure $d A_{\gamma}$ can be replaced by the measure $(1-|w|)^{\gamma} d A(w)$, as in [MS]. This results in the same space of functions and an equivalent norm, since $(1-|w|)^{\gamma}$ and $[\log (1 /|w|)]^{\gamma}$ are comparable for $1 / 2 \leq|w|<1$, and the singularity of $d A_{\gamma}$ at the origin is integrable.

The next results explain how the generalized counting functions $N_{\varphi, \gamma}$ arise naturally in the study of composition operators on Bergman and Hardy spaces. Let $\varphi$ be an analytic self-map of $D$, and recall from $\S 1$ the definition

$$
N_{\varphi, \gamma}(w)=N_{\gamma}(w)=\sum_{z \in \varphi^{-1}\{w\}}[\log (1 /|z|)]^{\gamma}, \quad w \in D \backslash\{\varphi(0)\} .
$$

This counting function provides the following non-univalent change of variable formula, due to Shapiro. 
2.2 Change of Variable Formula [Sh, p. 398]. If $g$ is a positive measurable function on $D$ and $\varphi$ is an analytic self-map of $D$, then

$$
\int_{D}(g \circ \varphi)\left|\varphi^{\prime}\right|^{2} d A_{\gamma}=c(\gamma) \int_{D} g N_{\varphi, \gamma} d A
$$

where $c(\gamma)=2^{\gamma} / \Gamma(\gamma+1)$.

The utility of this formula in the study of composition operators on Bergman and Hardy spaces comes from C. S. Stanton's formula for integral means of analytic functions [St]. We shall only require the following special case of this formula:

$$
\|f \circ \varphi\|_{H^{p}}^{p}=|f(\varphi(0))|^{p}+\frac{p^{2}}{2} \int_{D}|f(w)|^{p-2}\left|f^{\prime}(w)\right|^{2} N_{\varphi, 1}(w) d A(w) ;
$$

see (2.9) of [ESS]. This leads to an equivalent norm for $A_{\gamma}^{p}$ that will be very useful.

2.3 Lemma. If $0<p<\infty$, then

$$
\|f\|_{A_{\gamma}^{p}}^{p} \approx|f(0)|^{p}+\int_{D}|f(w)|^{p-2}\left|f^{\prime}(w)\right|^{2} d A_{\gamma+2}(w) .
$$

Here the symbol " $\approx$ " means that the left hand side is bounded above and below by constant multiples of the right hand side, where the constants are positive and independent of $f$.

Proof. First observe that we may assume $f(0)=0$, and then apply (2.1) with $\varphi(z)=r z$ to get

$$
\frac{1}{2 \pi} \int_{0}^{2 \pi}\left|f\left(r e^{i \theta}\right)\right|^{p} d \theta=\frac{p^{2}}{2} \int_{r D}|f(w)|^{p-2}\left|f^{\prime}(w)\right|^{2} \log \frac{r}{|w|} d A(w) .
$$

Next, multiply by $2 r[\log (1 / r)]^{\gamma}$, integrate with respect to $r$ for 0 to 1 , and then apply Fubini's theorem to get

$$
\begin{aligned}
\int_{D} \mid & \left.f(w)\right|^{p} d A_{\gamma}(w) \\
= & \frac{p^{2}}{2} \int_{0}^{1} \int_{D} \chi_{r D}(w)|f(w)|^{p-2}\left|f^{\prime}(w)\right|^{2} \log \frac{r}{|w|} d A(w) 2 r(\log (1 / r))^{\gamma} d r \\
= & \frac{p^{2}}{2} \int_{D}|f(w)|^{p-2}\left|f^{\prime}(w)\right|^{2}\left(\int_{|w|}^{1} 2 r[\log (1 / r)]^{\gamma} \log \frac{r}{|w|} d r\right) d A(w) .
\end{aligned}
$$

For $1 / 2 \leq|w|<1$, the inner integral with respect to $r$ is easily seen to be comparable to $[\log (1 /|w|)]^{\gamma+2}$. Since all singularities on the disk of radius $1 / 2$ are integrable, the inner integral can be replaced by $[\log (1 /|w|)]^{\gamma+2}$ without changing the space of functions for which it is finite, and an equivalent norm results.

In view of Lemma 2.3 and $(2.1)$ with $\varphi(z) \equiv z$, it is natural to define $A_{-1}^{p}$ to be the Hardy space $H^{p}$. Then

$$
\|f\|_{A_{\gamma}^{p}}^{p} \approx|f(0)|^{p}+\int_{D}|f(w)|^{p-2}\left|f^{\prime}(w)\right|^{2} d A_{\gamma+2}(w), \quad \gamma \geq-1,
$$

and we can give a unified treatment of the Hardy spaces and the weighted Bergman spaces. The following proposition is an immediate consequence of this norm estimate and the Change of Variable Formula 2.2. 
2.4 Proposition. Let $\varphi$ be an analytic self-map of $D$ and let $f$ be analytic on $D$. Then, for $\gamma \geq-1$,

$$
\|f \circ \varphi\|_{A_{\gamma}^{p}}^{p} \approx|f(\varphi(0))|^{p}+\int_{D}|f|^{p-2}\left|f^{\prime}\right|^{2} N_{\varphi, \gamma+2} d A .
$$

We will need the following simple growth estimate for a function in $A_{\alpha}^{p}$. It generalizes well known estimates for the functions in the classical Bergman and Hardy spaces, which correspond to $\alpha=0$ and $\alpha=-1$, respectively. In this lemma and in what follows, $C$ will denote a constant that can change from line to line, but is independent of $f$.

2.5 Lemma. Let $0<p<\infty$ and $\alpha \geq-1$. If $f \in A_{\alpha}^{p}$ and $w \in D$, then

$$
|f(w)| \leq C\|f\|_{A_{\alpha}^{p}}(1-|w|)^{-(\alpha+2) / p} .
$$

Proof. First note that, for $\alpha=-1, A_{-1}^{p}=H^{p}$ and this inequality is just the well known bound for the growth of a function in $H^{p}$; see Theorem 5.9, p. 84 of [D].

For $\alpha>-1$, the estimate for $|f|$ is a simple consequence of the subharmonicity of $|f|^{p}$ :

$$
\begin{aligned}
|f(w)|^{p} & \leq \frac{1}{A(D(w,(1-|w|) / 2))} \int_{D(w,(1-|w|) / 2)}|f|^{p} d A \\
& \leq C \frac{(1-|w|)^{-\alpha}}{A(D(w,(1-|w|) / 2))} \int_{D(w,(1-|w|) / 2)}|f|^{p} d A_{\alpha} \\
& \leq C \frac{\|f\|_{A_{\alpha}^{p}}^{p}}{(1-|w|)^{\alpha+2}} .
\end{aligned}
$$

\section{Sufficient CONDitions For $C_{\varphi}$ TO BE Bounded}

In this section we prove our main theorems giving sufficient conditions for $C_{\varphi}$ to be bounded. To simplify the notation, for the rest of the paper we will suppress the dependence on $\varphi$ of the generalized counting functions. Thus $N_{\gamma}=N_{\varphi, \gamma}$.

3.1 Theorem. Let $0<p \leq q$, and suppose $\varphi$ is an analytic self-map of $D$ satisfying

$$
N_{\beta+2}(w)=O\left([\log (1 /|w|)]^{(\alpha+2) q / p}\right) \quad(|w| \rightarrow 1) .
$$

Then

$$
C_{\varphi}: A_{\alpha}^{p} \rightarrow A_{\beta}^{q}
$$

is bounded.

Proof. By the Closed Graph Theorem, it suffices to show that $C_{\varphi}(f) \in A_{\beta}^{q}$ if $f \in A_{\alpha}^{p}$. Let $f \in A_{\alpha}^{p}$. Then, by Proposition 2.4,

$$
\|f \circ \varphi\|_{A_{\beta}^{q}}^{q} \approx|f(\varphi(0))|^{q}+\int_{D}|f|^{q-2}\left|f^{\prime}\right|^{2} N_{\beta+2} d A,
$$


and it is clear that $C_{\varphi}(f) \in A_{\beta}^{q}$ if and only if there is $r \in(0,1)$ such that

$$
\int_{D \backslash r D}|f|^{q-2}\left|f^{\prime}\right|^{2} N_{\beta+2} d A<\infty .
$$

By our hypothesis on the growth of $N_{\beta+2}$, there is a constant $K<\infty$ and $r_{0} \in(0,1)$ such that

$$
N_{\beta+2}(w) \leq K(\log (1 /|w|))^{(\alpha+2) q / p}, \quad w \in D \backslash r_{0} D .
$$

Using this and the growth estimate for $|f|$ from Lemma 2.5,

$$
\begin{aligned}
\int_{D \backslash r_{0} D}|f|^{q-2}\left|f^{\prime}\right|^{2} N_{\beta+2} d A & \\
& \leq C K\|f\|_{A_{\alpha}^{p}}^{q-p} \int_{D \backslash r_{0} D}|f(w)|^{p-2}\left|f^{\prime}(w)\right|^{2}(1-|w|)^{(p-q)(\alpha+2) / p} d A_{(2+\alpha) q / p}(w) .
\end{aligned}
$$

We may assume $r_{0} \geq 1 / 2$, so that $\log (1 /|w|) \leq 2(1-|w|)$. Using this estimate in our upper bound above, we see that

$$
\begin{aligned}
\int_{D \backslash r_{0} D}|f|^{q-2}\left|f^{\prime}\right|^{2} N_{\beta+2} d A & \leq C\|f\|_{A_{\alpha}^{p}}^{q-p} \int_{D \backslash r_{0} D}|f(w)|^{p-2}\left|f^{\prime}(w)\right|^{2} d A_{2+\alpha}(w) \\
& \leq C\|f\|_{A_{\alpha}^{p}}^{q},
\end{aligned}
$$

which completes the proof.

Recall from the remarks preceding Theorem 1.5 in the introduction that the sufficient condition in Theorem 3.1 for $C_{\varphi}$ to be bounded can not hold for all $p$ and $q$, although in the next section it will be shown that the condition is always necessary. The next theorem provides a sufficient condition for boundedness of $C_{\varphi}$ when $q<p$ that agrees with the condition in Theorem 3.1 in the limit as $q$ increases to $p$.

3.2 Theorem. Let $0<q<p$ and suppose $\varphi$ is an analytic self-map of $D$ satisfying

$$
N_{\beta+2}(w)=O\left([\log (1 /|w|)]^{\eta}\right) \quad(|w| \rightarrow 1),
$$

for some

$$
\eta>\frac{(\alpha+1) q+p}{p}
$$

Then

$$
C_{\varphi}: A_{\alpha}^{p} \rightarrow A_{\beta}^{q}
$$

is bounded.

Proof. Let $f \in A_{\alpha}^{p}$. As in the proof of Theorem 3.1, it suffices to show that $C_{\varphi}(f) \in A_{\beta}^{q}$, and for this it suffices to show there is $r \in(0,1)$ such that

$$
\int_{D \backslash r D}|f|^{q-2}\left|f^{\prime}\right|^{2} N_{\beta+2} d A<\infty .
$$


By our hypothesis on the growth of $N_{\beta+2}$, there is a constant $K<\infty$ and $r_{0} \in(0,1)$ such that

$$
N_{\beta+2}(w) \leq K(\log (1 /|w|))^{\eta}, \quad w \in D \backslash r_{0} D
$$

where

$$
\eta>\frac{(\alpha+1) q+p}{p}
$$

Now, $\alpha \geq-1$, and so $\eta>1$. Thus, by Lemma 2.3, it suffices to show that

$$
\int_{D \backslash r_{0} D}|f(w)|^{q}(1-|w|)^{\eta-2} d A(w)<\infty .
$$

By Hölder's inequality, this integral is bounded by

$$
\left(\int_{D}\left[|f(w)|^{q}(1-|w|)^{\alpha q / p}\right]^{p / q}\right)^{q / p}\left(\int_{D}\left[(1-|w|)^{\eta-2-\alpha q / p}\right]^{p /(p-q)} d A(w)\right)^{(p-q) / p} .
$$

The first factor is bounded by $\|f\|_{A_{\alpha}^{p}}^{q}$, and so is finite, while the second factor is finite because the assumed lower bound for $\eta$ is equivalent to the exponent in the integral being strictly greater than -1 . Thus the proof is complete.

\section{NeCESSARY CONDitions For $C_{\varphi}$ TO BE BOUnded}

The goal of this section is to give a necessary condition for $C_{\varphi}: A_{\alpha}^{p} \rightarrow A_{\beta}^{q}$ to be bounded. This condition matches the sufficient condition from Theorem 3.1 for $C_{\varphi}$ to be bounded when $q \geq p$, and so in this case there results a characterization of those $\varphi$ that induce bounded composition operators.

The proofs in this section are based on Shapiro's approach to finding a lower bound for the essential norm of a composition operator from $H^{2}$ to $H^{2}$, and from the weighted Bergman space $A_{\gamma}^{2}$ to itself. See sections 5 and 6 of [Sh], and Chapter 10 of [Sh2].

We begin with the statements of two lemmas concerning the generalized counting functions $N_{\gamma}$. The first lemma shows that these counting functions, while not subharmonic themselves, satisfy a subharmonic mean value property. It was first proved for the Nevanlinna counting function, $N_{1}$, in [ESS], section 6 . The version below, for the generalized counting functions, is from [Sh].

4.1 Lemma [Sh, Corollary 6.7]. Let $\psi$ be an analytic self-map of $D$ and let $\gamma>0$. If $\psi(0) \neq 0$ and $0<r<|\psi(0)|$, then

$$
N_{\psi, \gamma}(0) \leq \frac{1}{r^{2}} \int_{r D} N_{\psi, \gamma} d A .
$$

The next lemma shows how the counting functions transform under composition. The case $\gamma=1$ of this lemma can be found in [Sh], Chapter 10 . 
4.2 Lemma. Let $\psi$ be an analytic self-map of $D$, let $a \in D$ and let

$$
\sigma_{a}(w)=\frac{a-w}{1-\bar{a} w}
$$

be the Möbius self-map of $D$ that interchanges 0 and $a$. Then

$$
\left(N_{\psi, \gamma}\right) \circ \sigma_{a}=N_{\sigma_{a} \circ \psi, \gamma} .
$$

Proof. For each fixed $w \in \mathbb{C}, \psi-\sigma_{a}(w)$ and $\sigma_{a} \circ \psi-w$ have the same zero sequence, since $\sigma_{a}$ is its own compositional inverse. The result thus is an immediate consequence of the definition of the generalized counting function.

Next, we need to introduce a suitable class of test functions in $A_{\alpha}^{p}$ to establish the necessity of our conditions for the boundedness of the composition operators. To simplify the notation, the dependence of these functions on $\alpha$ and $p$ will be suppressed. For $a \in D$, define

$$
k_{a}(w)=\frac{\left(1-|a|^{2}\right)^{(2+\alpha) / p}}{(1-\bar{a} w)^{2(2+\alpha) / p}} .
$$

In the case that $\alpha=-1$, an easy computation involving power series shows that

$$
\left\|k_{a}\right\|_{A_{-1}^{p}}^{p}=\left(1-|a|^{2}\right)\left\|(1-\bar{a} w)^{-1}\right\|_{H^{2}}^{2}=1 .
$$

When $\alpha=0$, so $A_{0}^{p}$ is the unweighted Bergman space $A^{p}$, a similar computation again gives $\left\|k_{a}\right\|_{A^{p}}^{p}=1$. For other $\alpha>-1$, the computation is not so straightforward. Then

$\left\|k_{a}\right\|_{A_{\alpha}^{p}}^{p}=\left(1-|a|^{2}\right)^{2+\alpha} \int_{D} \frac{1}{|1-\bar{a} w|^{2(2+\alpha)}} d A_{\alpha}(w)=\left(1-|a|^{2}\right)^{2+\alpha}\left\|(1-\bar{a} w)^{-2-\alpha}\right\|_{A_{\alpha}^{2}}^{2}$.

As noted on page 400 of [Sh], a power series computation, using that $A_{\alpha}^{2}$ is a Hilbert space, now shows that

$$
\left\|k_{a}\right\|_{A_{\alpha}^{p}}^{p} \approx 1
$$

with constants depending only on $\alpha$ and $p$. This can also be seen by a straightforward estimate of the integral above over $D$. Thus approximation (4.1) holds for all $\alpha \geq-1$ and all $p>0$, since we previously saw that it holds for $\alpha=-1$.

4.3 Theorem. Suppose that $\varphi$ is an analytic self-map of $D$ that induces a bounded composition operator $C_{\varphi}: A_{\alpha}^{p} \rightarrow A_{\beta}^{q}$. Then

$$
N_{\beta+2}(w)=O\left((\log (1 /|w|))^{(2+\alpha) q / p}\right) \quad(|w| \rightarrow 1) .
$$

Proof. By Proposition 2.4, there is a constant $C_{1}$ such that

$$
\begin{aligned}
& C_{1}\left\|C_{\varphi}\left(k_{a}\right)\right\|_{A_{\beta}^{q}}^{q} \geq \int_{D}\left|k_{a}(w)\right|^{q-2}\left|k_{a}{ }^{\prime}(w)\right|^{2} N_{\beta+2}(w) d A(w) \\
&= \frac{4(2+\alpha)^{2}}{p^{2}}|a|^{2}\left(1-|a|^{2}\right)^{(2+\alpha) q / p} \int_{D} \frac{N_{\beta+2}(w)}{|1-\bar{a} w|^{2+2(2+\alpha) q / p}} d A(w) \\
&= \frac{4(2+\alpha)^{2}}{p^{2}}|a|^{2}\left(1-|a|^{2}\right)^{(2+\alpha) q / p-2} \int_{D} \frac{N_{\beta+2}(w)}{|1-\bar{a} w|^{2(2+\alpha) q / p-2}}\left|\sigma_{a}^{\prime}(w)\right|^{2} d A(w) \\
&= \frac{4(2+\alpha)^{2}}{p^{2}}|a|^{2}\left(1-|a|^{2}\right)^{(2+\alpha) q / p-2} \int_{D} \frac{N_{\beta+2}\left(\sigma_{a}(z)\right)}{\left|1-\bar{a} \sigma_{a}(z)\right|^{2(2+\alpha) q / p-2}} d A(z) .
\end{aligned}
$$


Here $\sigma_{a}=\sigma_{a}^{-1}$ is the Möbius self-map of $D$ that interchanges 0 and $a$, as in Lemma 4.2 , and the change of variable $z=\sigma_{a}(w)$ was made in the last line. Now,

$$
\frac{1}{\left|1-\bar{a} \sigma_{a}(z)\right|}=\frac{|1-\bar{a} z|}{1-|a|^{2}} \geq \frac{1}{2} \frac{1}{1-|a|^{2}}, \quad|z| \leq \frac{1}{2}
$$

and so

$$
C_{1}\left\|C_{\varphi}\left(k_{a}\right)\right\|_{A_{\beta}^{q}}^{q} \geq \frac{4^{2-(2+\alpha) q / p}(2+\alpha)^{2}|a|^{2}}{p^{2}\left(1-|a|^{2}\right)^{(2+\alpha) q / p}} \int_{\frac{1}{2} D} N_{\beta+2}\left(\sigma_{a}(z)\right) d A(z) .
$$

We now apply first Lemma 4.2, then Lemma 4.1 and then Lemma 4.2 again to see that, provided $\sigma_{a} \circ \varphi(0)>1 / 2$, the integral in the line above is at least

$$
\frac{1}{4} N_{\beta+2, \sigma_{a} \circ \varphi}(0)=\frac{1}{4} N_{\beta+2}(a) .
$$

Since $\sigma_{a} \circ \varphi(0)>1 / 2$ if $|a|$ is sufficiently close to 1 , this provides the estimate

$$
N_{\beta+2}(a) \leq C_{1}\left\|C_{\varphi}\left(k_{a}\right)\right\|_{A_{\beta}^{q}}^{q} \frac{4^{(2+\alpha) q / p-1} p^{2}}{(2+\alpha)^{2}|a|^{2}}\left(1-|a|^{2}\right)^{(2+\alpha) q / p}
$$

for all such $a$. Since $\left\|k_{a}\right\|_{A_{\alpha}^{p}} \approx 1$ and $\log (1 /|a|)$ is comparable to $\left(1-|a|^{2}\right)$ for $1 / 2<|a|<1$, the assumption that $C_{\varphi}$ is bounded provides the asserted bound for $N_{\beta+2}$, and the proof is complete.

As noted at the beginning of this section, the necessary condition in Theorem 4.3 for $C_{\varphi}$ to be bounded agrees with the sufficient condition from Theorem 3.1 that holds for $q \geq p$, and so the following corollary results.

4.4 Corollary. Let $0<p \leq q$ and let $\varphi$ be an analytic self-map of $D$. Then

$$
C_{\varphi}: A_{\alpha}^{p} \rightarrow A_{\beta}^{q}
$$

is bounded if and only if

$$
N_{\beta+2}(w)=O\left((\log (1 /|w|))^{(2+\alpha) q / p}\right) \quad(|w| \rightarrow 1) .
$$

Finally, we note that the parts of Theorem A and Theorem 1.1 of the introduction characterizing those $\varphi$ that induce bounded composition operators between Bergman and Hardy spaces are all special cases of Corollary 4.4. All that is required is to set $\alpha$ and $\beta$ equal to -1 or 0 , since $A_{-1}^{p}$ is the Hardy space $H^{p}$ and $A_{0}^{p}$ is the (unweighted) Bergman space $A^{p}$.

\section{Compactness of $C_{\varphi}$}

A bounded linear operator $T$ from a Banach space $X$ to a Banach space $Y$ is said to be compact provided the closure of $T(B)$ is a compact subset of $Y$, where $B$ is the unit ball of $X$. Equivalently, $T$ is compact if and only if some subsequence of $\left\{T\left(x_{n}\right)\right\}$ converges in $Y$, whenever $\left\{x_{n}\right\}$ is a bounded sequence in $X$.

In Corollary 4.4 of the last section, we saw that certain "big-oh" conditions were equivalent to some composition operators being bounded. The next theorem shows, as expected, that the corresponding "little-oh" conditions characterize compactness. Once again, the approach to the proof comes from [Sh]. See also Chapter 10 of [Sh2]. 
5.1 Theorem. Let $0<p \leq q$ and let $\varphi$ be an analytic self-map of $D$. Then

$$
C_{\varphi}: A_{\alpha}^{p} \rightarrow A_{\beta}^{q}
$$

is compact if and only if

$$
N_{\beta+2}(w)=o\left((\log (1 /|w|))^{(\alpha+2) q / p}\right) \quad(|w| \rightarrow 1) .
$$

Proof. We first show that $C_{\varphi}$ is compact, assuming that $N_{\beta+2}$ satisfies the given growth condition. Let $\left\|f_{n}\right\|_{A_{\alpha}^{p}} \leq 1$ for $n \geq 1$. We must show that $\left\{f_{n} \circ \varphi\right\}$ has a subsequence that converges in $A_{\beta}^{q}$. By Lemma 2.5, the $f_{n}$ are uniformly bounded on compact subsets of $D$, and hence $\left\{f_{n}\right\}$ is a normal family there. Thus there is a subsequence, which for simplicity we continue to denote by $\left\{f_{n}\right\}$, that converges uniformly on compact subsets of $D$ to an analytic function $f$. Now, for each $r \in(0,1)$,

$$
\int_{r D}|f|^{p-2}\left|f^{\prime}\right|^{2} d A_{\alpha+2}=\lim _{n \rightarrow \infty} \int_{r D}\left|f_{n}\right|^{p-2}\left|f_{n}\right|^{2} d A_{\alpha+2} \leq \limsup _{n \rightarrow \infty} C\left\|f_{n}\right\|_{A_{\alpha}^{p}} \leq C,
$$

by Lemma 2.3. It follows that $f \in A_{\alpha}^{p}$, and so $f \circ \varphi \in A_{\beta}^{q}$ by Theorem 3.1. To complete the proof, it suffices to show that $\left\|f_{n} \circ \varphi-f \circ \varphi\right\|_{A_{\beta}^{q}} \rightarrow 0$ as $n \rightarrow \infty$.

To establish this, note that from Proposition 2.4 it suffices to show that

$$
\begin{aligned}
& \left|f_{n}(\varphi(0))-f(\varphi(0))\right|^{p}+\int_{r D}\left|f_{n}-f\right|^{q-2}\left|f_{n}{ }^{\prime}-f^{\prime}\right|^{2} N_{\beta+2} d A \\
& +\int_{D \backslash r D}\left|f_{n}-f\right|^{q-2}\left|f_{n}{ }^{\prime}-f^{\prime}\right|^{2} N_{\beta+2} d A .
\end{aligned}
$$

can be made arbitrarily small by choosing $n$ large. For any fixed $r \in(0,1)$, the uniform convergence of $f_{n}$ to $f$ on compact subsets of $D$ shows that the first two terms in the display above converge to 0 as $n \rightarrow \infty$. Thus it suffices to show that the third term in (5.1) tends to zero, uniformly in $n$, as $r \rightarrow 0$. To this end, let $\epsilon>0$, and note that by hypothesis we can choose $r \in(0,1)$ so that

$$
\int_{D \backslash r D}\left|f_{n}-f\right|^{q-2}\left|f_{n}{ }^{\prime}-f^{\prime}\right|^{2} N_{\beta+2} d A \leq \epsilon \int_{D \backslash r D}\left|f_{n}-f\right|^{q-2}\left|f_{n}{ }^{\prime}-f^{\prime}\right|^{2} d A_{(\alpha+2) q / p} .
$$

We are now in exactly the same situation that occurred at the end of the proof of Theorem 3.1. Without providing all the details from that proof, the bound for $\left|f_{n}-f\right|$ from Lemma 2.5 leads to the estimate

$$
\int_{D \backslash r D}\left|f_{n}-f\right|^{q-2}\left|f_{n}{ }^{\prime}-f^{\prime}\right|^{2} N_{\beta+2} d A \leq \epsilon C\left\|f_{n}-f\right\|_{A_{\alpha}^{p}}^{q} \leq \epsilon C\left(\|f\|_{A_{\alpha}^{p}}+1\right)^{q} .
$$

Since $\epsilon>0$ was arbitrary, $C_{\varphi}$ is compact and the first part of the proof is complete.

We now finish the proof by assuming that $C_{\varphi}$ is compact and proving that $N_{\beta+2}$ satisfies the stated growth condition. For $a \in D$, let $k_{a}$ be as defined in $\S 4$,

$$
k_{a}(w)=\frac{\left(1-|a|^{2}\right)^{(2+\alpha) / p}}{(1-\bar{a} w)^{2(2+\alpha) / p}},
$$


and recall that $\left\|k_{a}\right\|_{A_{\alpha}^{p}} \approx 1$. Let $\left\{a_{n}\right\} \subset D$ satisfy $\left|a_{n}\right| \rightarrow 1$ as $n \rightarrow \infty$. From the definition of $k_{a}$, it is clear that this implies $k_{a_{n}}(z)$ converges uniformly to 0 on compact subsets of $D$ as $n \rightarrow \infty$. Hence the zero element of $A_{\beta}^{q}$ is the only possible limit point of $\left\{k_{a_{n}} \circ \varphi\right\}$. The compactness of $C_{\varphi}$ therefore yields that

$$
\lim _{|a| \rightarrow 1}\left\|C_{\varphi}\left(k_{a}\right)\right\|_{A_{\beta}^{q}}=0 .
$$

The required growth condition for $N_{\beta+2}$ is an immediate consequence of this and (4.2), the bound for $N_{\beta+2}$ that was derived in the proof of Theorem 4.3, and the proof is complete.

The nature of the "big-oh" sufficient condition in Theorem 3.2 for $C_{\varphi}$ to be bounded when $q<p$ is such that it is equivalent to the corresponding "little-oh" condition, and the same method used in the proof of Theorem 5.1 can be used to prove the next theorem. We omit the details.

5.2. Theorem. Let $0<q<p$ and suppose $\varphi$ is an analytic self-map of $D$ satisfying the conditions of Theorem 3.2. Then

$$
C_{\varphi}: A_{\alpha}^{p} \rightarrow A_{\beta}^{q}
$$

is compact.

We note that Theorem 1.5 of the introduction results from Theorems 3.2 and 5.2 by setting $\alpha=0$ and $\beta=-1$.

\section{Applications and Examples}

In this final section we present several examples and applications of our main results.

6.1 Finite valence maps. Let $\varphi$ be an analytic self-map of $D$. If $0<\sigma<\gamma$ and $w \in D \backslash\{\varphi(0)\}$, then

$$
N_{\gamma}(w) \leq\left(N_{\sigma}(w)\right)^{\gamma / \sigma} .
$$

This was proved in the introduction for $\sigma=1$ and $\gamma=2$, and the same proof works for any $\sigma<\gamma$. On the other hand, $N_{\sigma}$ can not in general be bounded by a multiple of a power of $N_{\gamma}$ when $\sigma<\gamma$. However, this is possible when $\varphi$ belongs to a large class of functions that includes the univalent functions.

Let $\varphi$ be an analytic self-map of $D$. We say $\varphi$ is of finite valence if there is an $M<\infty$ such that for all $w \in D$ the set $\varphi^{-1}\{w\}$ consists of at most $M$ points, counting multiplicity. Then, for $\sigma<\gamma$,

$$
\begin{aligned}
N_{\sigma}(w) & =\sum_{z \in \varphi^{-1}\{w\}}[\log (1 /|z|)]^{\sigma} \\
& \leq M \max _{z \in \varphi^{-1}\{w\}}[\log (1 /|z|)]^{\sigma} \\
& \leq M\left(\sum_{z \in \varphi^{-1}\{w\}}[\log (1 /|z|)]^{\gamma}\right)^{\sigma / \gamma} \\
& =M\left(N_{\gamma}(w)\right)^{\sigma / \gamma} .
\end{aligned}
$$


Combining this with (6.1), we see that if $\varphi$ has finite valence, then

$$
\left(N_{\sigma}(w)\right)^{\gamma} \approx\left(N_{\gamma}(w)\right)^{\sigma} .
$$

By setting $\sigma=1$ and $\gamma=2$ we therefore get the following corollary to Theorem A and Theorem 1.1 to add to those in the introduction.

6.2 Corollary. Let $\eta \geq 1$ and suppose $\varphi$ is an analytic self-map of $D$ having finite valence. Then the following are equivalent.

a) $C_{\varphi}: H^{p} \rightarrow H^{\eta p}$ is bounded for some (and hence all) $p>0$;

b) $C_{\varphi}: H^{p} \rightarrow A^{2 \eta p}$ is bounded for some (and hence all) $p>0$;

c) $C_{\varphi}: A^{p} \rightarrow A^{\eta p}$ is bounded for some (and hence all) $p>0$.

If $\eta \geq 2$, then the three statements above are in addition equivalent to

d) $C_{\varphi}: A^{p} \rightarrow H^{\eta p / 2}$ is bounded for some (and hence all) $p>0$.

Moreover, these remain equivalent when "bounded" is replaced by "compact".

Of course, statements b) and c) were previously shown to be equivalent even if $\varphi$ is not of finite valence (Corollary 1.3), as were a) and d) (Corollary 1.2). We also note that Corollary 4.4 can be combined with (6.2) for other choices of $\sigma$ and $\gamma$ to get similar results for other weighted Bergman spaces. We omit the details.

6.3 $C_{\varphi}: A^{p} \rightarrow H^{q}$ bounded for all $p$ and all $q$. We now turn to the construction of $\varphi$ that induce composition operators that are bounded from all the Bergman spaces to all the Hardy spaces. We saw a simple version of this in the introduction; $C_{\varphi}: A^{p} \rightarrow H^{\infty}$ is bounded if and only if $\varphi(D)$ is a relatively compact subset of $D$. We now want to construct more interesting examples for which $C_{\varphi}: A^{p} \rightarrow H^{q}$ is bounded for all $0<p \leq q<\infty$. In particular, in the introduction we promised an example of a univalent $\varphi$ satisfying this and in addition $\overline{\varphi(D)}=\bar{D}$.

The construction of this example requires some background on the hyperbolic metric. Let $\rho_{D}$ denote the hyperbolic metric on $D$, defined by (see [A, p. 2])

$$
\rho_{D}\left(z_{1}, z_{2}\right)=\inf \left\{\int_{\gamma} \frac{2|d z|}{1-|z|^{2}}: \gamma \text { is an arc in } D \text { from } z_{1} \text { to } z_{2}\right\} \text {. }
$$

We note for later use that a simple calculation shows

$$
\rho_{D}(0, z)=\log \frac{1+|z|}{1-|z|} .
$$

This distance is invariant under conformal self-maps of $D$, and therefore transfers to a natural conformally invariant metric on any simply connected proper subset $G \subset \mathbb{C}$. If $\varphi: D \rightarrow G$ is any conformal map, the hyperbolic distance on $G$ is given by $\rho_{G}\left(w_{1}, w_{2}\right)=\rho_{D}\left(z_{1}, z_{2}\right)$, where $w_{i}=\varphi\left(z_{i}\right)$ for $i=1,2$. However, $\rho_{G}\left(w_{1}, w_{2}\right)$ is not explicitly computable in terms of $G$ alone. A useful substitute is the quasi-hyperbolic metric on $G$, introduced by Gehring and Palka [GP]. For a domain $G \varsubsetneqq \mathbb{R}^{n}$ and $x \in G$, let $\delta_{G}(x)$ denote the Euclidean distance from $x$ to the complement of $G$. The quasi-hyperbolic distance from $x_{1}$ to $x_{2}$ in $G$ is defined to be

$$
k_{G}\left(x_{1}, x_{2}\right)=\inf \left\{\int_{\gamma} \frac{d s}{\delta_{G}(x)}: \gamma \text { is an arc in } G \text { from } x_{1} \text { to } x_{2}\right\} .
$$


Here $d s$ denotes integration with respect to arclength.

The quasi-hyperbolic metric is closely related to the hyperbolic metric. Indeed, if $G \varsubsetneqq \mathbb{C}$ is a simply connected domain, then

$$
\frac{1}{2} \rho_{G} \leq k_{G} \leq 2 \rho_{G} .
$$

See, for example, [Sh2, Chapter 9] for these inequalities and further background on the hyperbolic metric. Due to the geometric nature of its definition, $k_{G}$ is very useful in obtaining estimates for the hyperbolic metric.

We now return to the promised example, beginning with the proof of a theorem that gives a simple geometric condition that is sufficient for $C_{\varphi}$ to have the required properties. Recall that $\delta_{G}(w)$ is the Euclidean distance from $w$ to $\mathbb{C} \backslash G$ for $w \in G$, and define $\delta_{G}(w)=0$ if $w \in \mathbb{C} \backslash G$.

6.4 Theorem. Let $G \subset D$ be a simply connected domain such that

$$
\lim _{|w| \rightarrow 1} \frac{\delta_{G}(w)}{1-|w|}=0
$$

If $\varphi: D \rightarrow G$ is a Riemann map, then $C_{\varphi}: A^{p} \rightarrow H^{q}$ is bounded for all $0<p \leq$ $q<\infty$.

Proof. Suppose $G \subset D$ satisfies the given hypotheses and $\varphi: D \rightarrow G$ is a Riemann map. We may assume that $0 \in G$ and $\varphi(0)=0$. Since $\varphi$ is univalent, it follows immediately from the definition of $N_{\varphi, 1}$ that

$$
N_{\varphi, 1}(w)=O\left([\log (1 /|w|)]^{\eta}\right) \quad(|w| \rightarrow 1)
$$

if and only if

$$
1-|z|=O\left((1-|\varphi(z)|)^{\eta}\right) \quad(|z| \rightarrow 1) .
$$

Therefore, by Theorem $1.1 \mathrm{~b}), C_{\varphi}: A^{p} \rightarrow H^{q}$ will be bounded for all $0<p \leq q<\infty$ if and only if (6.6) is satisfied for all $\eta>0$.

By conformal invariance of the hyperbolic metric and (6.3), we see that (6.6) is equivalent to

$$
\exp \left(-\rho_{G}(0, \varphi(z))\right)=\exp \left(-\rho_{D}(0, z)\right)=O\left(\exp \left(-\eta \rho_{D}(0, \varphi(z))\right)\right) \quad(|z| \rightarrow 1) .
$$

Thus, taking logarithms and setting $\varphi(z)=w$, we must show that for a given $\eta>0$ there exists a constant $C$ such that

$$
\eta \rho_{D}(0, w) \leq C+\rho_{G}(0, w), \quad w \in G .
$$

Let $\eta>0$ be given and, by $(6.5)$, choose $r \in(0,1)$ such that $4 \eta \delta_{G}(z) \leq 1-|z|$ if $|z|>r$ and $z \in G$. Now set $C=\eta \rho_{D}(0, r)$. Then (6.7) is clearly satisfied if $|w| \leq r$, so assume $|w|>r$. Then, using (6.4),

$$
\eta \rho_{D}(0, w)=C+\eta \rho_{D}(r,|w|) \leq C+2 \eta k_{D}(r,|w|)=C+2 \eta \int_{r}^{|w|} \frac{d t}{1-t} .
$$


Now, for $\gamma$ any arc in $G$ from 0 to $w$,

$$
\int_{r}^{|w|} \frac{d t}{1-t} \leq \int_{\gamma \cap(D \backslash r D)} \frac{d s}{4 \eta \delta_{G}(z)} \leq \int_{\gamma} \frac{d s}{4 \eta \delta_{G}(z)},
$$

by the choice of $r$. Taking the infimum over all such arcs $\gamma$, appending the resulting estimate to the preceding inequality, and then using (6.4) again, we get

$$
\eta \rho_{D}(0, w) \leq C+2 \eta \int_{r}^{|w|} \frac{d t}{1-t} \leq C+\frac{1}{2} k_{G}(0, w) \leq C+\rho_{G}(0, w) .
$$

Thus (6.7) is satisfied and the proof is complete.

With this theorem in hand, it is easy to produce the promised example.

6.5 Example. There exists a univalent $\varphi: D \rightarrow D$ such that

a) $C_{\varphi}: A^{p} \rightarrow H^{q}$ is bounded for all $0<p \leq q<\infty$ and

b) $\overline{\varphi(D)}=\bar{D}$.

To see this, let $G_{0}=D$ and, proceeding by induction, assume that $n \geq 0$ and $G_{n}$ has been constructed by deleting finitely many radial slits from $D$ so that

$$
\delta_{G_{n}}(w) \leq 3^{-n}, \quad \text { if } w \in G_{n} \text { and } 1-|w| \leq 2^{-n} .
$$

We then construct $G_{n+1}$ by deleting from $G_{n}$ finitely many radial slits (with one endpoint on $\partial D$ ) of length $2^{-n-1}$ so that (6.8) is satisfied with $n$ replaced by $n+1$. With $G_{n}$ now defined for all $n \geq 0$, set $G=\bigcap_{n \geq 0} G_{n}$. From (6.8) and the construction it is clear that $\delta_{G}(w) \leq 3^{-n}$ if $2^{-n-1}<1-|w| \leq 2^{-n}$, so that (6.5) is satisfied. Hence if $\varphi: D \rightarrow G$ is a Riemann map, then Theorem 6.4 applies and $C_{\varphi}: A^{p} \rightarrow H^{q}$ is bounded for all $0<p \leq q<\infty$. Since in addition $\overline{\varphi(D)}=\bar{G}=\bar{D}$, $\varphi$ has all the required properties and the example is complete.

6.6 Polygonal maps. We now present a class of self-maps of $D$ for which the hypotheses of the main theorems of this paper are easily verified. Let $P \subset \bar{D}$ be a polygon, and let $\psi$ be a Riemann map from $D$ onto the interior of $P$. We then call $\psi$ a polygonal self-map of $D$. The composition operators induced by such functions have been studied by several authors, including Shapiro and Taylor in [ST] and Riedl in $[R]$. In particular, the first conclusion in the theorem below can be derived from Theorem V.1 in $[R]$, which was proved using different methods.

The case that $P$ is a compact subset of $D$ is not interesting, since then $C_{\psi}: A^{p} \rightarrow$ $H^{\infty}$ is bounded for all $p>0$, as was observed in the introduction. So suppose $P$ has a vertex at $w=1$ with an associated angle of $\pi / \eta$, and $\alpha$ is the minimal angle the two adjacent sides of $P$ make with the tangent line to $\partial D$ at $w=1$. Thus $\eta>1$ and $\alpha>0$. As is well known, $\psi$ extends to be a homeomorphism of $\bar{D}$ onto $P$, and we assume $\psi(1)=1$. By the Schwarz reflection principle,

$$
\frac{(\psi(z)-1)^{\eta}}{z-1}
$$

extends to be analytic and non-zero in a neighborhood of $z=1$. Thus

$$
1-|z| \leq|1-z| \leq C|1-\psi(z)|^{\eta} \leq C(\csc (\alpha))^{\eta}(1-|\psi(z)|)^{\eta}
$$

in a neighborhood of $z=1$. These observations make it easy to prove the next theorem. 
6.7 Theorem. Let $P \subset \bar{D}$ be a polygon and let $\pi / \eta$ be the largest angle at a vertex of $P$ that lies on $\partial D$. If $\varphi$ is an analytic self-map of $D$ with $\varphi(D) \subset P$, then

$$
C_{\varphi}: H^{p} \rightarrow H^{\eta p} \quad \text { and } \quad C_{\varphi}: A^{p} \rightarrow A^{\eta p}
$$

are bounded for all $p>0$. If in addition $\eta \geq 2$, then

$$
C_{\varphi}: A^{p} \rightarrow H^{\eta p / 2}
$$

is bounded for all $p>0$. In each case above, the conclusion is sharp in the sense that $C_{\varphi}$ need not be compact and moreover the exponent in the target space can not be increased. Finally, if $2>\eta>(p+q) / p$ (or equivalently $q<(\eta-1) p<p)$, then

$$
C_{\varphi}: A^{p} \rightarrow H^{q}
$$

is bounded, and moreover is compact.

Proof. Let $\psi$ be the polygonal self-map of $D$ with $\psi(\bar{D})=P$. Since $\pi / \eta$ is the largest angle at the finitely many vertices of $P$ on $\partial D$, we have by (6.9) that

$$
1-|z|=O\left((1-|\psi(z)|)^{\eta}\right) \quad(|z| \rightarrow 1)
$$

and so (see (6.6))

$$
N_{\psi, 1}(w)=O\left([\log (1 /|w|)]^{\eta}\right) \quad(|w| \rightarrow 1)
$$

since $\psi$ is univalent. Also, by (6.1),

$$
N_{\psi, 2}(w)=O\left([\log (1 /|w|)]^{2 \eta}\right) \quad(|w| \rightarrow 1) .
$$

Thus, from Theorem A, Theorem 1.1 and Theorem 1.5, the conclusions of the theorem are satisfied when $C_{\varphi}$ is replaced by $C_{\psi}$.

Now, since $\psi$ is univalent and $\varphi(D) \subset \psi(D), \varphi$ is subordinate to $\psi$. That is, there is a self-map $\sigma$ of $D$ such that $\varphi=\psi \circ \sigma$, or $C_{\varphi}=C_{\sigma} \circ C_{\psi}$. Recall that $C_{\sigma}: H^{q} \rightarrow H^{q}$ and $C_{\sigma}: A^{q} \rightarrow A^{q}$ are bounded for all $q>0$ by Littlewood's subordination principle, and so the result follows.

To see that the results are sharp, note that when $z=r$ is real the quantities in (6.9) are all comparable. Thus the exponent $\eta$ in (6.10) can not be increased, and moreover the corresponding "little-oh" condition fails. Since $\psi$ is univalent, we have by (6.2) that the same applies to (6.11). Theorem A and Theorem 1.1 therefore show that the conclusions of the theorem are sharp for $C_{\psi}$.

Remark. Under the hypotheses of Theorem 6.7 we also have that $C_{\varphi}: H^{p} \rightarrow A^{2 \eta p}$ is bounded. This can be derived from Theorem 1.1 and (6.11) as above. However, it is also a direct consequence of the first conclusion of Theorem 6.7, since $H^{\eta p} \subset A^{2 \eta p}$ (see the remarks following Corollary 1.4), and so it was left out of the statement. 


\section{REFERENCES}

[A] Ahlfors, L.V., Conformal Invariants, McGraw-Hill, New York, 1973. MR 50:10211

[D] Duren, P.L., Theory of $H^{p}$ Spaces, Academic Press, New York, 1970. MR 42:3552

[ESS] Essén, M., Shea, D.F. and Stanton, C.S., A value-distribution criterion for the class $L \log L$, and some related questions, Ann. Inst. Fourier (Grenoble) 35 (1985), 127-150. MR 87e:30041

[GP] Gehring, F. W. and Palka, B. P., Quasiconformally homogeneous domains, J. Analyse Math. 30 (1976), 172-199. MR 55:10676

[H] Hunziker, H., Kompositionsoperatoren auf klassischen Hardyräumen, Thesis, Universität Zurich (1989).

[HJ] Hunziker, H. and Jarchow, H., Composition operators which improve integrability, Math. Nachr. 152 (1991), 83-91. MR 93d:47061

[L] Littlewood, J.E., On inequalities in the theory of functions, Proc. London Math. Soc. 23 (1925), 481-519.

[MS] MacCluer, B.D. and Shapiro, J.H., Angular derivatives and compact composition operators on the Hardy and Bergman spaces, Canad. J. Math. 38 (1986), 878-906. MR 87h:47048

[R] Riedl, R., Composition operators and geometric properties of analytic functions, Thesis, Universität Zurich (1994).

[Sh] Shapiro, J.H., The essential norm of a composition operator, Annals of Math. 127 (1987), 375-404. MR 88c: 47058

[Sh2] Shapiro, J.H., Composition Operators and Classical Function Theory, Springer-Verlag, New York Berlin, 1993. MR 94k:47049

[ST] Shapiro, J.H. and Taylor, P.D., Compact, nuclear and Hilbert-Schmidt composition operators on $H^{2}$, Indiana University Math. J. 23 (1973), 471-496. MR 48:4816

[St] Stanton, C. S., Counting functions and majorization for Jensen measures, Pacific J. Math. 125 (1986), 459-468. MR 88c:32002

Department of Mathematics, University of Hawail, Honolulu, Hawail 96822

E-mail address: wayne@math.hawaii.edu 